\title{
Silicon carbide quantum dots for bioimaging
}

\author{
David Beke ${ }^{\text {a) }}$ \\ Institute for Solid State Physics and Optics, Wigner Research Centre for Physics, Hungarian Academy of Sciences, \\ H-1121 Budapest, Hungary; and Faculty of Chemical Technology and Biotechnology, Budapest University of \\ Technology and Economics, H-1111 Budapest, Hungary \\ Zsolt Szekrényes \\ Institute for Solid State Physics and Optics, Wigner Research Centre for Physics, Hungarian Academy of Sciences, \\ H-1121 Budapest, Hungary \\ Denes Pálfi \\ The Faculty of Information Technology, Pázmány Péter Catholic University, H-1083 Budapest, Hungary; and \\ Institute of Experimental Medicine, Hungarian Academy of Sciences, H-1083 Budapest, Hungary
}

Gergely Róna

Institute of Enzymology, Research Centre of Natural Sciences, Hungarian Academy of Sciences, H-1113 Budapest, Hungary

István Balogh

Institute for Solid State Physics and Optics, Wigner Research Centre for Physics, Hungarian Academy of Sciences, H-1121 Budapest, Hungary

Pal Andor Maák

Department of Atomic Physics, Budapest University of Technology and Economics, H-1111 Budapest, Hungary

Gergely Katona

Institute of Experimental Medicine, Hungarian Academy of Sciences, H-1083 Budapest, Hungary

Zsolt Czigány

Institute for Technical Physics and Materials Science, Research Centre of Natural Sciences, Hungarian Academy of Sciences, H-1121 Budapest, Hungary

Katalin Kamarás

Institute for Solid State Physics and Optics, Wigner Research Centre for Physics, Hungarian Academy of Sciences, H-1121 Budapest, Hungary

Balazs Rózsa

The Faculty of Information Technology, Pázmány Péter Catholic University, H-1083 Budapest, Hungary; and Institute of Experimental Medicine, Hungarian Academy of Sciences, H-1083 Budapest, Hungary

Laszlo Buday and Beata Vértessy

Institute of Enzymology, Research Centre of Natural Sciences, Hungarian Academy of Sciences, H-1113 Budapest, Hungary

Adam Gali

Institute for Solid State Physics and Optics, Wigner Research Centre for Physics, Hungarian Academy of Sciences, H-1121 Budapest, Hungary; and Department of Atomic Physics, Budapest University of Technology and Economics, H-1111 Budapest, Hungary

(Received 30 May 2012; accepted 10 August 2012)

Luminescent nanocrystals or quantum dots (QDs) have great potential for bioanalysis as well as optoelectronics. Here we report an effective and inexpensive fabrication method of silicon carbide quantum dots ( $\mathrm{SiC}$ QDs), with diameter below $8 \mathrm{~nm}$, based on electroless wet chemical etching. Our samples show strong violet-blue emission in the $410-450 \mathrm{~nm}$ region depending on the solvents used and particle size. The cytotoxic properties of the SiC QDs based on alamarBlue ${ }^{\mathrm{TM}}$ assay cells were studied. The presence of the QDs dots does not affect cell growth in a wide concentration range. Two-photon excitation showed significant response from $\mathrm{SiC}$ nanocrystals that were injected into hippocampal CA1 pyramidal cells.

\footnotetext{
a) Address all correspondence to this author.

e-mail: beke.david@wigner.mta.hu

DOI: $10.1557 /$ jmr.2012.296
}

\section{INTRODUCTION}

The present revolution in basic and applied biological research, like drug delivery or bioimaging techniques, strongly depends on the availability of nanosized crystals 
with adequate attributes linked especially to their nontoxic and bioinert properties. ${ }^{1}$ Semiconductor quantum dots (QDs) are emerging as alternative or complementary tools to organic fluorescent dyes, which are currently used in bioimaging. In comparison with traditional organic fluorophores, QDs have a number of advantages including broad excitation and narrow emission spectra. QDs are more resistant to photobleaching than their organic counterparts, making QDs superior alternative materials for biomarkers and for further applications in biology and medicine. $^{2}$

QDs are often made of group II and VI elements [e.g., cadmium selenide ( $\mathrm{CdSe})$ and cadmium telluride $(\mathrm{CdTe})]^{3}$ or group III and V elements [e.g., indium phosphide (InP) and indium arsenide (InAs)]. ${ }^{4}$ Although these QDs have great potential as probes for bioimaging, certain limitations may restrict their applications. These QDs were found to be cytotoxic through the release of free metallic ions and therefore a protective shell must be systematically added. However no protective shell can guarantee an efficient chemical isolation of the extremely toxic elements from the living cellular environment. Cytotoxicity is one of the major limiting factors when applying II-VI QDs in in vivo imaging. ${ }^{5}$

Luminescent silicon ( $\mathrm{Si}$ ) QDs have great potential in biological imaging and diagnostic applications. ${ }^{6}$ For exploiting this potential, they must remain luminescent and stably dispersed in water and biological fluids over a wide range of $\mathrm{pH}$ and salt concentration. There are many challenges in creating such stable water-dispersible Si QDs, as they show strong instability in the photoluminescence properties due to their fast oxidation in aqueous environments. Another difficulty is related to the attachment of hydrophilic molecules to the Si QDs' surfaces. Oxide surface passivation is due to either a $\mathrm{Si}=\mathrm{O}$ double bond or a Si-O-Si ether-like bond. ${ }^{6,7}$ Attaching organic ligands to the surface of Si QDs is an alternative way to passivate the Si surface. A large family of organic molecules can be grafted to the surface of Si QDs, resulting in Si-C surfacebond capping. ${ }^{7,8}$ The strong Si-C bonds prevent the surface from being oxidized and these Si-based QDs are watersoluble at the same time. $\mathrm{Si}-\mathrm{C}$ bonds are naturally present in silicon carbide that can provide a stable surface per se.

In this study, we propose silicon carbide ( $\mathrm{SiC}$ ) QDs as a promising material to be used in biomarker- and bioimaging-related applications. SiC QDs were successfully fabricated in many ways. ${ }^{9-12}$ The typical diameter is often less than $5 \mathrm{~nm}$. Small size is also of great importance in living cell applications for clearance. In addition, biocompatibility of bulk $\mathrm{SiC}^{13,14}$ and $\mathrm{SiC}$ QDs has been already proven in the literature. ${ }^{15,16}$ The $\mathrm{Si}-\mathrm{C}$ bonds in $\mathrm{SiC}$ add chemical stability to $\mathrm{SiC}$ QDs, thus there is no need for surface passivation after synthesis and they form stable colloids in water. The presence of oxidized carbon groups on the surface of $\mathrm{SiC}$ QDs opens new possibilities for easy biofunctionalization and derivatization processes for further applications. ${ }^{17}$

We use electroless wet chemical etching ${ }^{18}$ of the $\mathrm{SiC}$ microcrystals to form nanosize SiC QDs. Photoluminescence properties and surface termination of our SiC QDs have already been described. ${ }^{17}$ In this article, we investigate their cytotoxicity and demonstrate their potential in bioimaging techniques by using two-photon microscopy for capturing images from SiC QD-injected pyramidal cells.

\section{EXPERIMENT}

\section{A. Preparation of the SiC QDs}

Silicon powder (365 mesh) and graphite powder were the starting materials as silicon and carbon source. A silicongraphite mixture with 1:1.2 M ratio was pressed into a pellet, then placed into an induction chamber $(400 \mathrm{kHz})$ and heated up to about $1300{ }^{\circ} \mathrm{C}$ under argon atmosphere where the reaction began. The residual graphite was removed by annealing the sample at $900{ }^{\circ} \mathrm{C}$ for $10 \mathrm{~h}$ under air and the residual $\mathrm{Si}$ was eliminated by using a hydrofluoric acid (HF) and nitric acid $\left(\mathrm{HNO}_{3}\right)$ mixture at room temperature. In the second step the electroless wet chemical etching method was performed which results in the formation of a highly porous layer on the $\mathrm{SiC}$ microcrystal (SiC MC) surfaces. The acid mixture used for the etching procedure was $65 \% \mathrm{HNO}_{3}: 48 \%$ $\mathrm{HF}$ with a volume ratio of 1:3, the etching was performed at $100{ }^{\circ} \mathrm{C}$ for $2 \mathrm{~h}$. After removing the acids and washing the samples the SiC QDs were obtained by sonication of the porous $\mathrm{SiC}$ MCs in water or in ethanol for $40 \mathrm{~min}$ to remove the porous layer and suspend the QDs. The $\mathrm{SiC}$ $\mathrm{MCs}$, cleaned from the porous layer, and the $\mathrm{SiC}$ QDs were separated by centrifugation at $2600 \mathrm{~g}$ for $1.5 \mathrm{~h}$. The supernatant fluid contained SiC QDs.

\section{B. Characterization of the SiC QDs}

Figure 1 shows a typical high-resolution transmission electron microscope image (HRTEM; JEOL JEM-3010, $300 \mathrm{kV}$, Tokyo, Japan; $1.7 \AA$ resolution) of SiC QDs and their size distribution. The HRTEM image reveals that the SiC QDs are nearly spherical and the typical lattice spacing of $0.25 \mathrm{~nm}$ corresponds to the (111) plane of 3C-SiC. The size distribution was obtained from several HRTEM images. The typical size was $\sim 3 \mathrm{~nm}$ with relatively small dispersions in size $(1-8 \mathrm{~nm})$.

Photoluminescence (PL) of the SiC QDs was studied in water and ethanol solution in the $300-400 \mathrm{~nm}$ excitation range using a Horiba Jobin-Yvon Nanolog fluorometer (Horiba Scientific, Edison, NJ). The highest band intensity appears at an excitation of $360-370 \mathrm{~nm}$ with the corresponding emission in the range of 420-450 nm (Fig. 2 for the aqueous colloidal solution).

The surface structure of the SiC QDs was characterized by infrared spectroscopy (Bruker Tensor 37 FTIR spectrometer, Bruker Optik GmbH, Ettlingen, Germany, 
equipped with an overhead attenuated total reflectance unit for liquids). ${ }^{17}$ Our study revealed $\mathrm{Si}-\mathrm{O}-\mathrm{Si}, \mathrm{C}-\mathrm{O}-\mathrm{C}$, $\mathrm{CH}, \mathrm{COOH}$, and $\mathrm{COO}^{-}$groups on the $\mathrm{SiC}$ QDs surfaces. Thanks to these polar and/or ionic groups on the surface, $\mathrm{SiC}$ QDs make stable colloids in water and open new possibilities for further functionalization processes.

\section{Growth inhibition assay}

The immortal HeLa cell lines pertaining to human cervical adenocarcinoma were purchased from the major cell-line depository, American Type Culture Collection (ATCC). The cells were cultured in Dulbecco's Modified Eagle Medium (DMEM)/ F12 HAM nutrient mixture (Sigma-Aldrich Co. LLC, St. Louis, MI) supplemented with Penicillin-Streptomycin solution (Gibco; $50 \mathrm{mg} / \mathrm{mL}$;) and $10 \%$ fetal bovine serum (FBS) (GIBCO Products International, Inc., Langley, OK). Cell viability was measured by alamarBlue ${ }^{\mathrm{TM}}$ Cell Viability Assay Kit (Biotium, Inc., Hayward, CA). Exponentially growing cells were plated in an equal number (4000 cells/well) onto 96-well

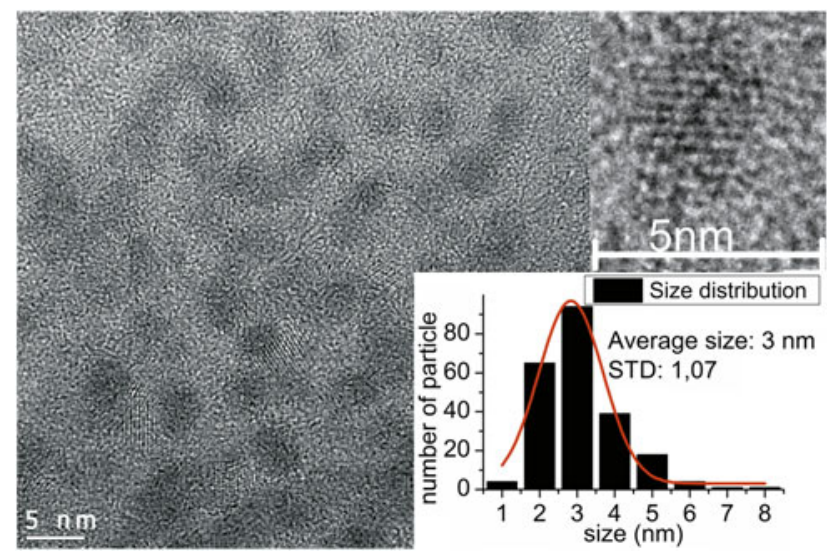

FIG. 1. HRTEM image and size distribution of the SiC QDs distributed on an amorphous carbon foil.

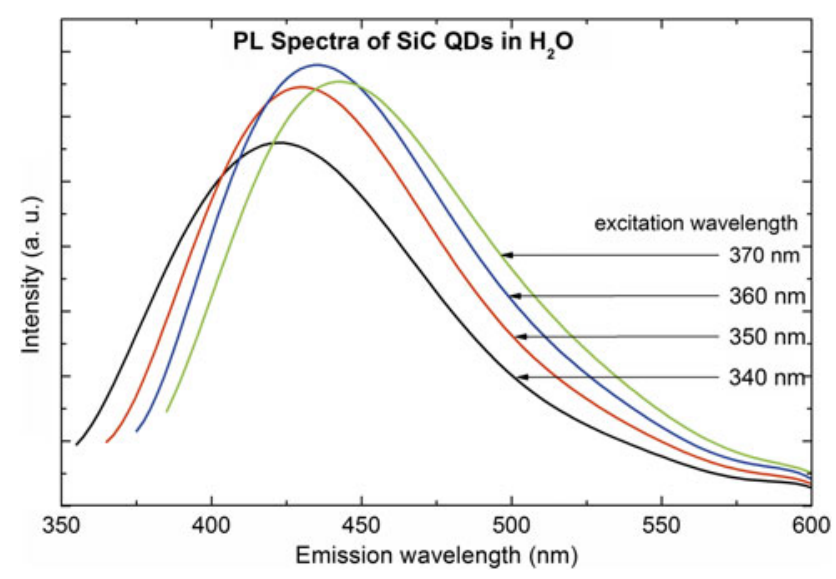

FIG. 2. PL spectra of SiC QDs in water. tissue culture plates. The cells were exposed for $48 \mathrm{~h}$ to increasing concentrations of $\mathrm{SiC}$ QDs. After $48 \mathrm{~h}$, alamarBlue ${ }^{\mathrm{TM}}$ solution was added to each well at $10 \%(\mathrm{v} / \mathrm{v})$ and the cells were further incubated for $3 \mathrm{~h}$. The resulting fluorescence was measured using $530 \mathrm{~nm}$ excitation and $590 \mathrm{~nm}$ emission wave lengths in a microtiter plate-reader WallacVictor-2 (Perkin Elmer, Waltham, MA). IC50 values were defined as the applied SiC QD concentration $(\mu \mathrm{M})$ required for half-maximal inhibitory effect on cell growth. IC50 values were calculated by nonlinear curve-fitting using Origin Lab 8.5 software. Experiments were performed in triplicate and standard deviation values were calculated (Fig. 3)

Based on our growth inhibition assay, it could be seen that $\mathrm{SiC}$ QDs created in this study do not affect cell growth in a wide concentration range. They can be applied even up to $100 \mu \mathrm{g} / \mathrm{mL}$ in the medium without significant effect on cell survival for $48 \mathrm{~h}$. This implies the possible use of such nanocrystals for live cell labeling techniques.

Botsoa et al. ${ }^{15}$ and Serdiuk et al. ${ }^{16}$ demonstrated that $\mathrm{SiC}$ QDs formed by means of electrochemical anodization of a bulk $\mathrm{SiC}$ polycrystalline wafer were able to penetrate through the cell membrane. In our similar experiments, we did not find any specific uptake mechanism: there were no differences between the samples treated by SiC QDs and the reference sample except for higher background fluorescence from the SiC QD treated sample (not shown). The contradiction between previous results ${ }^{15,16}$ and the ones presented here may stem from the preparation method: in our electroless wet chemical etching method we apply an oxidizing agent, $\mathrm{HNO}_{3}$, that could result in different surface compared with the case of previous experiments based on electrochemical etching where only $\mathrm{HF}$ and ethanol are applied as solvents. This property of our SiC QDs may be advantageous in controlling their future functionalization for tracking exclusively the targeted biomolecules.

\section{Two-photon microscopy}

Our PL measurements indicate a strong excitation in the green wave length region upon appropriate excitation energy (see Fig. 2). As the excitation wave length moves toward $400 \mathrm{~nm}$, the emission shows a small red shift. For

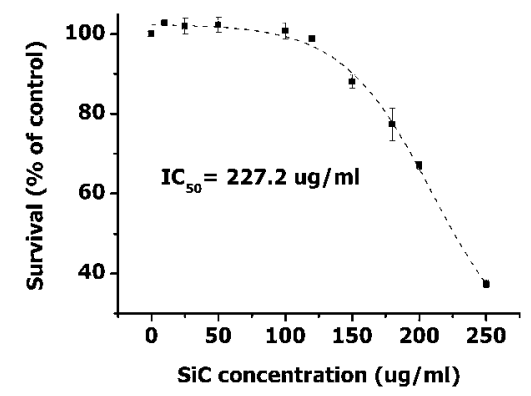

FIG. 3. Cell viability measurement results and calculated IC50 value for $\mathrm{SiC}$ QDs by the use of AlamarBlue assay. 
in vivo studies red or near-infrared region excitation and emission are desirable. Two-photon excitation of SiC QDs may work efficiently for in vivo bioimaging because short near-infrared pulses can penetrate deeply in organic tissues (unlike photons in the ultraviolet or visible region), and our SiC QDs absorb single photons in the ultraviolet region, which corresponds to absorption in the near-infrared region by two-photon processes.

Indeed, we demonstrate here that our $\mathrm{SiC}$ QDs have a strong emission due to two-photon excitation. We detected fluorescence from $\mathrm{SiC}$ QDs injected to neuron cells by a two-photon microscope. Two-photon imaging was performed using a Femto2D Two-Photon Laser Scanning Microscope (2PLSM; Femto2D, Femtonics Ltd., Budapest, Hungary) with an ultrafast Ti:Sapphire laser (Mai Tai, Spectra Physics) tuned from 740 to $850 \mathrm{~nm} .{ }^{19}$ Optimal excitation wave length for imaging in red channel (emission wave lengths between 600 and $700 \mathrm{~nm}$ ) was around $830 \mathrm{~nm}$. The excitation laser energy was $\sim 60 \mathrm{~mW}$ before the objective.

Whole cell patch-clamp recordings were made from CA1 pyramidal neurons in acute $300 \mu \mathrm{m}$ mouse hippocampal slices. Cells were filled with intracellular solution (ICs) and SiC QDs (concentration of $0.5 \mathrm{mg} / \mathrm{mL}$ ) via the patch-pipette (6-9 M $\Omega$ ). Images were taken at $\sim 40 \mu \mathrm{m}$ under the slice surface. Cells in the middle of CA1 stratum pyramidale were identified as pyramidal cells by video microscopy using oblique infrared illumination and by analyzing their response to somatic current injections, serving also as a measure of cell viability [Fig. 4(c)]. The neuronal cell body, soma, was clearly visible in the red channel, and even the apical dendrite near to the soma could be resolved [Fig. 4(b)]. However the signal contrast was relatively weak in the green channel [425-525 nm, Fig. 4(a)], which is a bit unexpected since the maximum emission of $\mathrm{SiC}$ QDs in water is in the green channel by single photon excitation. Possibly, the altered ion and protein concentrations might generate a change in the emission spectra and fluorescent intensity as it also occurs in case of other fluorescent dyes. ${ }^{20}$ Further investigations are needed to clear this issue. Z-stacks of red channel images were also taken confirming homogeneous loading of soma and proximal dendrites [Fig. 4(d)]. As we expected from the bioinert property of SiC QDs, no sign of cellular damage of the pyramidal neurons was observable during the long $(>1 \mathrm{~h})$ measurement duration. In addition, basic electrophysiological properties (action potential threshold, peak width and amplitude; membrane potential; firing rate) have not changed significantly during the measurement indicating the lack of cellular toxicity. This study implies that our SiC QDs may be good candidates as luminescent biomarkers for neuroscience.

\section{SUMMARY}

In summary, well-defined spherical colloidal cubic $\mathrm{SiC}$ nanocrystals with average diameter of $3 \mathrm{~nm}$ were synthesized using the reactive bonding and inexpensive wet chemical etching method. Based on our growth inhibition

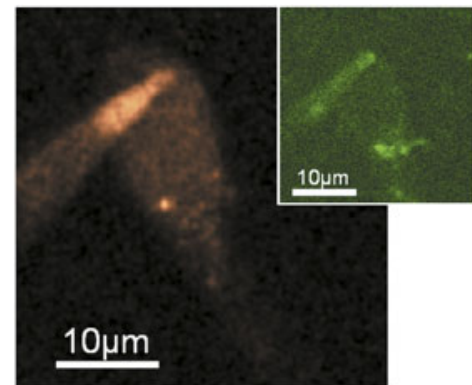

(a)

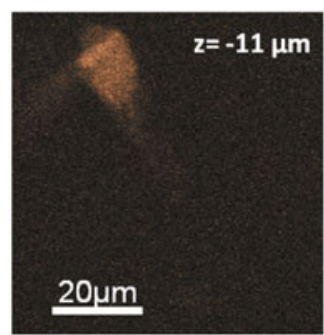

(d)

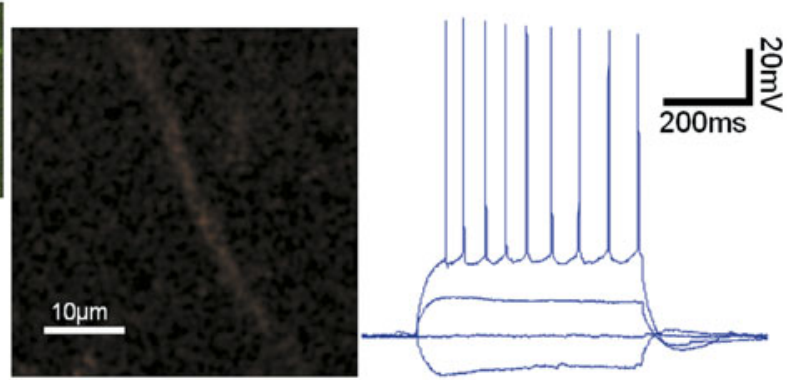

(b)
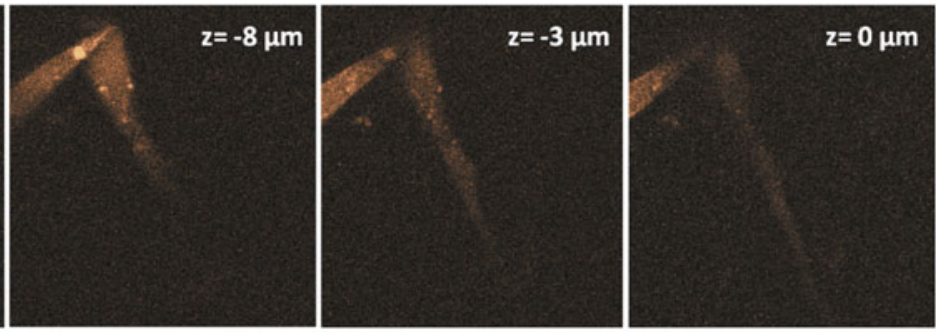

(c)

FIG. 4. Two-photon imaging of a neuron labeled by SiC QDs. (a) Representative 2PLSM image of a CA1 hippocampal pyramidal neuron filled with $\mathrm{SiC}$ QDs. Red fluorescence signal (600-700 nm, red channel) was generated at $830 \mathrm{~nm}$ excitation. Inset, the same neuron but fluorescent signal was collected from 425 to $525 \mathrm{~nm}$ (green channel). (b) Red channel image of the apical dendrite of the neuron. (c) Somatic membrane voltage responses of the neuron induced by somatic current injection show normal functioning of the neuron. (d) 2PLSM images collected at different depths using $800 \mathrm{~nm}$ excitation. 
assay it could be seen that $\mathrm{SiC}$ nanocrystals created in this study do not affect cell growth in a wide concentration range. They can be applied even up to $100 \mu \mathrm{g} / \mathrm{mL}$ in the cell medium without significant effect on cell survival for $48 \mathrm{~h}$. This implies the possibility of use of such nanocrystals safely for live cell labeling techniques. We demonstrate that $\mathrm{SiC}$ fluorophores are feasible candidates for two-photon in vitro bioimaging.

\section{ACKNOWLEDGMENTS}

Hungarian OTKA Grant No. 101819 and Bolyai János Research Scholarship of HAS for Zs.C. are acknowledged. A.G. and L.B. acknowledge the support from the special research program "Lendület" from the Hungarian Academy of Sciences. Two-photon imaging was supported by Hungarian-French Grant (TÉT_101-2011-0389), Hungarian-Swiss Grant (SH/7/2/8), and OTKA (K 105997). Hungarian OTKA Grant Nos. 106114 and 106216 are also acknowledged.

\section{REFERENCES}

1. M.J. Murcia and C.A. Naumann: Biofunctionalization of fluorescent nanoparticles, in Nanotechnologies for Life Sciences, Vol. 1, edited by S.S.R. Kumar (Wiley-VCH, Weinheim, 2005), pp. 1-40.

2. I.L. Medintz, H.T. Uyeda, E.R. Goldman, and H. Mattoussi: Quantum dot bioconjugates for imaging, labeling and sensing. Nat. Mater. 4, 435, (2005).

3. S. Kim, B. Fisher, H.J. Eisler, and M. Bawendi: Type-II quantum dots: $\mathrm{CdTe} / \mathrm{CdSe}$ (core/shell) and $\mathrm{CdSe} / \mathrm{ZnTe}$ (core/shell) heterostructures. J. Am. Chem. Soc. 125, 11466, (2003).

4. Y.W. Cao, J. Aksenton, V. Soloviev, and U. Banin: Colloidal synthesis and properties of InAs/InP and InAs/CdSe core/shell, nanocrystals. in Semiconductor Quantum Dots, edited by S.C. Moss, D. Ila, H.W.H. Lee, and D.J. Norris (Mater. Res. Soc. Symp. Proc. 571, Warrendale, PA, 2000) p. 75.

5. R. Hardman: A toxicologic review of quantum dots: Toxicity depends on physicochemical and environmental factors. Environ. Health Perspect. 114, 165, (2006).

6. F. Hua, F. Erogbogbo, K.T. Yong, I. Roy, G.X. Xu, P.N. Prasad, and M.T. Swihart: Organically capped silicon nanoparticles with blue photoluminescence prepared by hydrosilylation followed by oxidation. ACS Nano 2, 873, (2008).
7. Y. Kanemitsu, N. Shimizu, T. Komoda, P.L.F. Hemment, and B.J. Sealy: Photoluminescent spectrum and dynamics of $\mathrm{Si}^{+}$. ion-implanted and thermally annealed $\mathrm{SiO}_{2}$ glasses. Phys. Rev. B 54, R14329 (1996).

8. G. Hadjisawas and P. Kelires: Structure and energetics of Si nanocrystals embedded in a-SiO ${ }_{2}$. Phys. Rev. Lett. 93, 226104 (2004).

9. X. Wu, J. Fan, T. Qiu, X. Yang, G. Siu, and P.K. Chu: Experimental evidence for the quantum confinement effect in 3C-SiC nanocrystallites. Phys. Rev. Lett. 94, 6 (2005).

10. J.Y. Fan, X.L. Wu, H.X. Li, H.W. Liu, G.G. Siu, and P.K. Chu: Luminescence from colloidal $3 \mathrm{C}-\mathrm{SiC}$ nanocrystals in different solvents. Appl. Phys. Lett. 88, 041909 (2006).

11. J. Botsoa, J.M. Bluet, V. Lysenko, O. Marty, D. Barbier, and G. Guillot: Photoluminescence of $6 \mathrm{H}-\mathrm{SiC}$ nanostructures fabricated by electrochemical etching. J. Appl. Phys. 102, 083526 (2007).

12. Z. Makkai, B. Pécz, I. Bársony, G. Vida, A. Pongrácz, K.V. Josepovits, and P. Deák: Isolated $\mathrm{SiC}$ nanocrystals in $\mathrm{SiO}_{2}$. Appl. Phys. Lett. 86, 253109 (2005).

13. C. Coletti, M.J. Jaroszeski, A. Pallaoro, A.M. Hoff, S. Iannotta, and S.E. Saddow: Biocompatibility and wettability of crystalline SiC and Si surfaces. In IEEE EMBS Proceedings 29th Annual International Conference. 5849-5852 (EMBS, Lyon, France, 2007).

14. C.T. Raya, D.H. Maldonado, J.R. Rico, C.G. Gañan, A.R. de Arellano-Lopez, and J.M. Fernandez: Fabrication, chemical etching, and compressive strength of porous biomimetic $\mathrm{SiC}$ for medical implants. J. Mater. Res. 23, 3247-3254 (2008).

15. J. Botsoa, V. Lysenko, A. Géloën, O. Marty, J.M. Bluet, and G. Guillot: Application of 3C-SiC quantum dots for living cell imaging. Appl. Phys. Lett. 92, 173902 (2008).

16. T. Serdiuk, V. Lysenko, V. Skryshevsky, and A. Géloën: Vapor phase-mediated cellular uptake of sub-5 nm nanoparticles. Nanoscale Res. Lett. 7, 212 (2012).

17. D. Beke, Zs. Szekrényes, I. Balogh, M. Veres, É. Fazakas, L.K. Varga, K. Kamarás, Zs. Czigány, and A. Gali: Characterization of luminescent silicon carbide nanocrystals prepared by reactive bonding and subsequent wet chemical etching. Appl. Phys. Lett. 99, 213108 (2011)

18. J. Zhu, Z. Liu, X.L. Wu, L.L. Xu, W.C. Zhang, and P.K. Chu: Luminescent small-diameter $3 \mathrm{C}-\mathrm{SiC}$ nanocrystals fabricated via a simple chemical etching method. Nanotechnology 18, 365603 (2007).

19. G. Katona, G. Szalay, P. Maák, A. Kaszás, M. Veress, D. Hillier, B. Chiovini, E.S. Vizi, B. Roska, and B. Rózsa: Fast two-photon in vivo imaging with three-dimensional random-access scanning in large tissue volumes. Nat. Methods 9, 201 (2012).

20. M. Maravall, Z.F. Mainen, B.L. Sabatini, and K. Svoboda: Estimating intracellular calcium concentrations and buffering without wavelength ratioing. Biophys. J. 78, 2655-2667 (2000). 